\title{
Neonatal basal ganglia and thalamic volumes: very preterm birth and 7-year neurodevelopmental outcomes
}

\author{
Wai Yen Loh ${ }^{1}$, Peter J. Anderson ${ }^{2}$, Jeanie L.Y. Cheong ${ }^{3}$, Alicia J. Spittle ${ }^{4}$, Jian Chen ${ }^{5}$, Katherine J. Lee ${ }^{6}$, \\ Charlotte Molesworth ${ }^{7}$, Terrie E. Inder ${ }^{8}$, A. Connelly ${ }^{9}$, Lex W. Doyle ${ }^{10}$ and Deanne K. Thompson ${ }^{7}$
}

BACKGROUND: This study aims to (i) compare volumes of individual basal ganglia nuclei (caudate nucleus, pallidum, and putamen) and the thalamus between very preterm (VP) and term-born infants at term-equivalent age; (ii) explore neonatal basal ganglia and thalamic volume relationships with 7-year neurodevelopmental outcomes, and whether these relationships differed between VP and term-born children.

METHODS: 210 VP ( $<30$ weeks' gestational age) and 39 term-born ( $\geq 37$ weeks' gestational age) infants underwent brain magnetic resonance imaging at term-equivalent age, and deep gray matter volumes of interest were automatically generated. 186 VP and 37 term-born children were assessed for a range of neurodevelopmental measures at age 7 years. RESULTS: All deep gray matter structures examined were smaller in VP infants compared with controls at termequivalent age; ranging from (percentage mean difference (95\% confidence intervals) $-6.2 \%(-10.2 \%,-2.2 \%)$ for the putamen, to $-9.5 \%(-13.9 \%,-5.1 \%)$ for the caudate nucleus. Neonatal basal ganglia and thalamic volumes were positively related to motor, intelligence quotient, and academic outcomes at age 7 years, with mostly similar relationships in the VP and control groups.

CONCLUSION: VP birth results in smaller basal ganglia and thalamic volumes at term-equivalent age, and these smaller volumes are related to a range of 7-year neurodevelopmental deficits in VP children.

V ery preterm (VP; <32 weeks' gestational age) infants are at an increased risk for later impairments across cognitive, academic, motor, and behavioral domains compared with their term-born ( $\geq 37$ weeks' gestational age) peers $(1,2)$. VP birth occurs at a critical time in brain development, and altered brain structure in this population is associated with poorer neurodevelopmental outcomes in VP survivors (3). However, the specific neuroanatomic correlates of neurodevelopmental impairments in VP children remain unclear.

The basal ganglia and thalamus are deep gray matter structures of the brain, critical for modulation of a wide range of motor, cognitive, and behavioral functions (4). The basal ganglia comprise a complex of nuclei, the major nuclei being the caudate nucleus, putamen, nucleus accumbens (collectively termed striatum), and pallidum. As brain relay structures, the basal ganglia and thalamus form parallel functional circuits with the cortex (5), whereby the striatum receives input from cortical areas subserving cognitive, sensorimotor, and affective functions, and relays the information to the pallidum. The pallidum then outputs the information to the thalamus, which closes the circuit by projecting back to the cortex.

Few studies have investigated basal ganglia and thalamic volumes in VP infants $(3,6,7)$. Of those, two studies have attempted to examine the effect of VP birth on individual basal ganglia nuclei $(6,7)$ but measured the putamen and pallidum as a combined structure (lentiform nuclei). Despite close neuroanatomical proximity, these two nuclei are separate and have different hierarchical positions within the organization of the basal ganglia and thalamic circuits (5). The current study builds on previous research by examining the effect of VP birth on the individual components that are uniquely positioned within the basal ganglia and thalamic circuits.

There has been limited research examining the relationships between neonatal deep gray matter volumes and long-term neurodevelopmental outcomes in preterm children. Preterm studies have predominantly reported neonatal deep gray matter volume relationships with neurodevelopmental outcomes in early childhood (1-4 years) $(3,8,9)$. Early developmental outcomes, however, are only moderately predictive of longer-term outcomes (10). Only one preterm study has examined longer-term outcomes, reporting a relationship between deep gray matter volume at term-equivalent age and

\footnotetext{
${ }^{1}$ The Florey Department of Neuroscience and Mental Health, University of Melbourne, Melbourne, Victoria, Australia; ${ }^{2}$ Monash Institute of Cognitive and Clinical Neurosciences, Monash University, Clayton, Victoria, Australia; ${ }^{3}$ Department of Obstetrics and Gynecology, University of Melbourne, Melbourne, Victoria, Australia; ${ }^{4}$ Department of Physiotherapy, University of Melbourne, Melbourne, Victoria, Australia; ${ }^{5}$ Department of Medicine, Stroke and Ageing Research Group, Southern Clinical School, Monash University, Melbourne, Victoria, Australia; ${ }^{6}$ Department of Pediatrics, University of Melbourne, Melbourne, Victoria, Australia; ${ }^{7}$ Murdoch Childrens Research Institute, Melbourne, Victoria, Australia; ${ }^{8}$ Brigham and Women's Hospital, Boston, Massachusetts; ${ }^{9}$ Florey Institute of Neuroscience and Mental Health, Melbourne, Victoria, Australia; ${ }^{10}$ Neonatal Services, Royal Women's Hospital, Melbourne, Victoria, Australia. Correspondence: Wai Yen Loh (waiyen.loh@mcri.edu.au)

Received 29 December 2016; accepted 12 May 2017; advance online publication 30 August 2017. doi:10.1038/pr.2017.161
} 
motor impairment at age 11 years (11). These previous studies $(3,8,9,11)$ did not incorporate a comparison group, explored a limited range of neurodevelopmental outcomes, and only one study (9) examined individual neonatal deep gray matter volumes. The current study overcomes these limitations by investigating individual deep gray matter structures and examining a broad range of neurodevelopmental outcomes in a large and representative cohort of VP and term-born 7-yearolds.

Using a new automated segmentation pipeline for pediatric brain magnetic resonance imaging (MRI), the aims of the current study were to (i) compare volumes of individual basal ganglia nuclei (caudate nucleus including the nucleus accumbens, pallidum, and putamen) and the thalamus between VP and term-born controls at term-equivalent age and (ii) explore neonatal basal ganglia and thalamic volume relationships with 7-year neurodevelopmental outcomes (intelligence quotient (IQ), academic achievement, executive functioning, motor, and behavior), and whether these relationships differed between VP and term-born children. We hypothesized that VP infants would have smaller basal ganglia and thalamic volumes compared with controls, and that reduced basal ganglia and thalamic volumes would be associated with worse neurodevelopment in both VP and control groups.

\section{METHODS}

\section{Participants}

All participants were recruited at the Royal Women's Hospital in Melbourne, Australia as part of the prospective longitudinal Victorian Infant Brain Studies cohort between July 2001 and December 2003. Two hundred and twenty-four VP infants ( $<30$ weeks' gestational age and/or $<1,250 \mathrm{~g}$ birth weight) and 46 term-born infants (37-42 weeks' gestational age and $\geq 2,500 \mathrm{~g}$ birth weight) without congenital abnormalities were eligible for the study and underwent brain MRI at term-equivalent age (target scan period: 38-42 weeks' gestational age, median scan age: 40 weeks' gestational age). Participants were followed up at age 7 years with a neuropsychological assessment undertaken at the Murdoch Childrens Research Institute, Melbourne, Australia. The study was approved by the Human Research Ethics Committees at the Royal Women's and Royal Children's Hospitals. Parents gave written informed consent for child's participation.

\section{MRI Analysis}

Infants were scanned using a 1.5 Tesla General Electric Signa MRI scanner at the Royal Children's Hospital. Infants were fed, swaddled, placed in a vacuum-fixation bean bag, fitted with earmuffs, and scanned while sleeping without sedation. $\mathrm{T}_{2}$-weighted and proton density-weighted dual-echo fast recovery spin echo sequences were acquired interleaved $(1.7-3.0 \mathrm{~mm}$ coronal slices; repetition time $4,000 \mathrm{~ms}$; echo time $60 / 160 \mathrm{~ms}$; flip angle $90^{\circ}$; field of view $220 \times 160 \mathrm{~mm}^{2}$; matrix $256 \times 192$; interpolated $\left.512 \times 512\right)$. The $\mathrm{T}_{2}$-weighted and proton density-weighted images were combined to improve contrast in the deep gray matter (12), resampled into $0.43 \mathrm{~mm}$ isotropic voxels, bias-corrected, and brain-extracted.

Volumetric measurements of the basal ganglia and thalamus were obtained following a previously described Pediatric Subcortical Segmentation Technique (PSST) (13), which recommends the construction of a normative structural MRI template with basal ganglia and thalamic tissue priors to accurately segment these structures in a pediatric brain. Following PSST, a representative neonatal structural MRI template was constructed with Advanced Normalization Tools (ANTs) $(14,15)$ from the MR images of five VP infants (26.6-28.4 weeks' gestational age; two males and three females) and five term-born infants (38.1-42.0 weeks' gestational age; three males and two females), who were from a separate study that acquired higher quality $\mathrm{T}_{2}$-weighted transverse restore turbospin echo sequences on a 3 Tesla Trio Siemens MRI machine $(1 \mathrm{~mm}$ isotropic voxels; repetition time $8,910 \mathrm{~ms}$; echo time $152 \mathrm{~ms}$; flip angle $120^{\circ}$; field of view $192 \times 192 \mathrm{~mm}^{2}$; and matrix $192 \times 192$ ) $(16,17)$. To create the neonatal basal ganglia and thalamic tissue priors, the thalamus, pallidum, and putamen were manually traced as separate structures on the $10 \mathrm{MR}$ images. The caudate nucleus and nucleus accumbens were manually traced as a complex (CNAcc) on the $10 \mathrm{MR}$ images because the nucleus accumbens could not be discriminated as a distinct structure at this early age. Intrarater reliability was conducted on the 10 de-identified MR images, by manually tracing a second time 1 week later. The Dice coefficient was high ( $>0.89$ for all structures), indicating reliable manual tracing of the basal ganglia and thalamus. The basal ganglia and thalamic

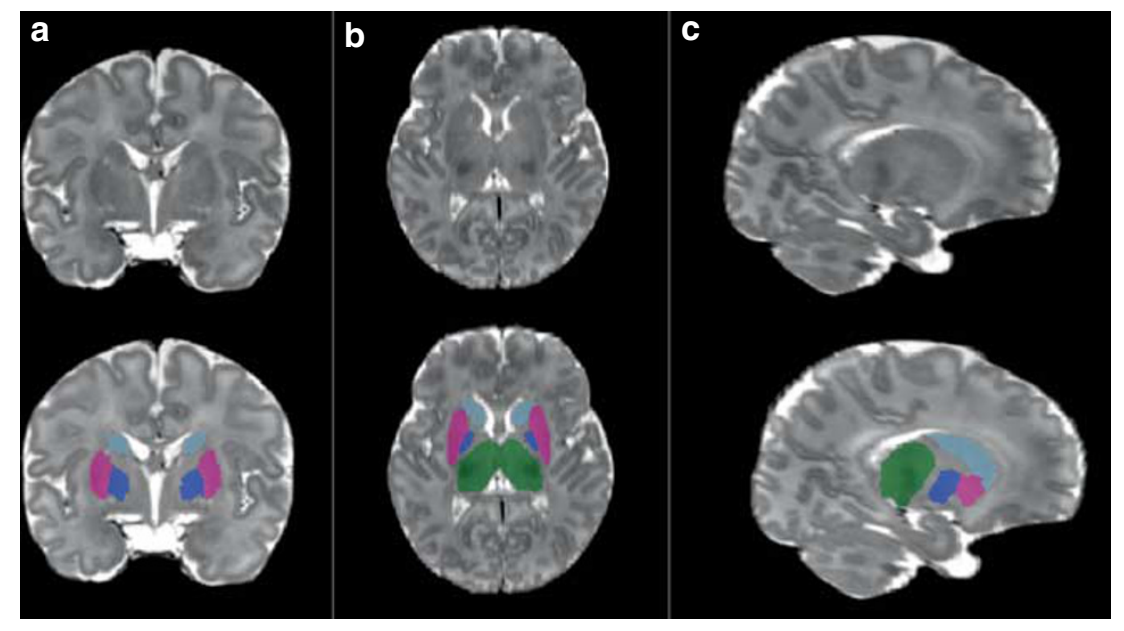

Figure 1. Basal ganglia and thalamus segmentation of a term-born infant using combined $T_{2}$-weighted and proton density-weighted MR images, shown in (a) coronal, (b) axial, and (c) sagittal views. The colors represent the segmented basal ganglia and thalamic structures as follows: light blue = caudate nucleus (includes the nucleus accumbens); dark blue= pallidum; fuchsia = putamen; and green=thalamus. 
manual tracings of the 10 infants were brought into the neonatal MRI template space and averaged together to create tissue priors.

Application of the neonatal basal ganglia and thalamic tissue priors to the participants' MRI data was done following the PSST pipeline. The other tissue priors required for segmentation with PSST included cerebrospinal fluid, which was obtained from the Morphologically Adaptive Neonatal Tissue Segmentation (MANTiS) tool (18), and the cortex, white matter, cerebellum, and brainstem, all of which were derived from the 40-week infant template developed by the Biomedical Image Analysis Group, Imperial College London (19). All tissue priors were registered to each participant's MR image with ANTs (14). Segmentation was performed in native space using the Unified Segmentation tool in the Statistical Parametric Mapping (SPM) software (20). Post-processing was done with MATLAB 2010b to morphologically fill in holes within the basal ganglia and thalamus segmentation labels. All segmentation outputs (see Figure 1 for example) were inspected. One term-born and thirteen VP infants were manually edited for obvious errors by W.Y.L.

Intracranial volume (ICV), which comprised total cerebrospinal fluid volume and total brain tissue volume, was obtained using MANTiS (18).

\section{Neuropsychological Assessment}

At age 7 years, children were assessed by psychologists and pediatricians blinded to MRI findings and clinical history, including preterm birth.

General cognitive ability was measured using full-scale IQ, as estimated by the Wechsler Abbreviated Scale of Intelligence (mean $(M)=100$, standard deviation $(\mathrm{SD})=15)(21)$.

Basic educational skills including word reading and math computation were assessed by the Wide Range Achievement Test 4 $(M=100, \mathrm{SD}=15)(22)$.

Executive function was assessed across three domains (attentional control, working memory, and goal setting). (i) Attentional control was assessed using the Score! subtest (auditory sustained attention) from the Test of Everyday Attention for Children $(M=10, S D=3)$ (23). (ii) Working memory was assessed using the Backward Digit Recall from the Working Memory Test Battery for Children $(M=100, \mathrm{SD}=15)(24)$. (iii) Goal setting was assessed with the Tower of London, with performance judged using a total summary score (25).

Motor skills were evaluated using the Movement Assessment Battery for Children Second Edition (MABC-2), which is a reliable and valid measure of gross and fine motor skills including aiming and catching, manual dexterity, and postural control based upon normative data from the United Kingdom $(M=10, \mathrm{SD}=3)(26)$.

Behavior was evaluated with a parent report questionnaire, the Strengths and Difficulties Questionnaire (SDQ), and the total behavioral score was used (maximum score $=50)(27)$.

A composite measure of social risk was determined by a questionnaire at 7 years of age, based on family structure, education of the primary caregiver, occupation and employment status of the primary income earner, dominant language spoken in the home, and maternal age at birth (28). Each component of social risk was scored on a 3-point scale (zero representing the lowest risk and 2 representing the highest risk), and summed to give a total score (range, 0-12).

\section{Statistical Analysis}

All statistical analyses were performed using Stata 14.0. Participant characteristics were compared between those who did and did not have analyzable MRI data using $t$-tests, Mann-Whitney $U$-tests, or $\chi^{2}$ tests.

Group differences in basal ganglia and thalamic volumes at termequivalent age were assessed using separate linear regression models for each structure, with sex and age at MRI scan included in the model as potential confounders. Volumes from the two hemispheres were combined for each structure, as there has been little evidence that the effect of VP birth on the cross-sectional area of deep gray matter structures differs between hemispheres (29). Results are presented as the percentage volume difference (95\% confidence intervals (CIs)) of the VP group compared with the control group. A sensitivity analysis was performed excluding VP infants with cystic periventricular leukomalacia (PVL) and/or severe intraventricular hemorrhage (grades 3 and 4).

Neonatal basal ganglia and thalamic volume associations with neurodevelopmental outcomes at age 7 years were explored using separate linear regression models for each volume-outcome combination per structure. A group-by-volume interaction term was included in the model to examine whether the effect of structure volume on outcome measure varied by group. Sex, social risk, and age at assessment were also included as potential confounders. Results are presented as coefficients (95\% CI) for every $10 \%$ increase in structure volume. Percentage change in the brain volume was calculated relative to the mean volume in the control group for the particular structure. This aids comparisons of the volume-outcome relationships between structures, which have different absolute raw volumes. Volume-outcome relationships were plotted for all participants if the group-by-volume interaction term has a $P$-value $>0.05$; otherwise, the relationships were plotted separately for the VP and control groups.

All linear regression models were fitted using generalized estimating equations and robust standard errors to allow for the clustering of multiple births. All analyses were repeated adjusted for ICV to examine whether this explained any of the group differences or volume-outcome relationships. Given the multiple comparisons, results were interpreted as overall patterns and magnitudes of relationships instead of focusing on individual $P$-values, consistent with modern statistical practice.

\section{RESULTS}

\section{Sample Characteristics}

Fourteen (6.3\%) VP and 7 (15.2\%) term-born children were excluded from analyses due to movement artifact in the MR images, leaving $210 \mathrm{VP}$ and 39 term-born children for analysis. Of these, $186 \mathrm{VP}$ and 37 term-born children had 7year neurodevelopmental assessments. As expected, the VP group differed from the control group on most of the perinatal variables, and the VP group performed more poorly than the control group for most of the neurodevelopmental measures at age 7 years (Table 1). Similar perinatal characteristics were observed between those who had usable and unusable MRI data at term-equivalent age.

\section{Basal Ganglia and Thalamic Volumes: VP vs. Term Controls} Mean basal ganglia and thalamic volumes are shown separately for the VP and control groups in Figure 2a. VP infants had smaller volumes across all basal ganglia and thalamic structures at term-equivalent age compared with controls (Figure 2b). The percentage volume difference (coefficient $(95 \% \mathrm{CI})$ ) ranged from $-6.2 \%(-10.2 \%,-2.2 \%)$ for putamen to $-9.5 \%(-13.9 \%,-5.1 \%)$ for CNAcc. Findings of volumetric group differences remained after adjustment for ICV. In the sensitivity analysis excluding VP infants with PVL and/or moderate-to-severe intraventricular hemorrhage (grades 3 and 4), VP infants without severe brain lesions still had smaller basal ganglia and thalamic volumes compared with controls at term-equivalent age (Supplementary Material S1 online). 
Table 1. Perinatal and 7-year characteristics of the study's participants

\begin{tabular}{lcc}
\hline Perinatal characteristics & VP $(n=210)$ & Controls $(n=39)$ \\
\hline $\begin{array}{l}\text { Gestational age at birth } \\
\text { (weeks), } M(\mathrm{SD})\end{array}$ & $27.5(1.9)$ & $38.9(1.3)$ \\
Birth weight (g), $M(\mathrm{SD})$ & $959(227)$ & $3269(496)$ \\
Birth weight SD score, $M(\mathrm{SD})$ & $-0.57(0.95)$ & $0.06(0.89)$ \\
Singleton, $n$ (\%) & $124(59.0)$ & $37(94.9)$ \\
Male, $n$ (\%) & $108(51.4)$ & $20(51.3)$ \\
Antenatal corticosteroids, $n$ (\%) & $186(89.0)$ & $0(0)$ \\
Postnatal corticosteroids, $n(\%)$ & $19(9.1)$ & $0(0)$ \\
Infection, $n$ (\%) & $84(40.0)$ & $1(2.6)$ \\
Oxygen dependency at & $71(33.8)$ & $0(0)$ \\
36 weeks, $n$ (\%) & &
\end{tabular}

White matter injury severity, $\mathrm{n}(\%)^{\mathrm{a}}$

\begin{tabular}{|c|c|c|}
\hline None & $91(43.3)$ & $33(84.6)$ \\
\hline Mild & $77(36.7)$ & $6(15.4)$ \\
\hline Moderate & $29(13.8)$ & - \\
\hline Severe & $11(5.2)$ & - \\
\hline $\begin{array}{l}\text { Cystic periventricular } \\
\text { leukomalacia, } n(\%)\end{array}$ & $9(4.3)$ & $0(0)$ \\
\hline $\begin{array}{l}\text { Intraventricular hemorrhage } \\
\text { grades } 3 / 4, n(\%)\end{array}$ & $7(3.3)$ & $0(0)$ \\
\hline $\begin{array}{l}\text { Age at MRI (gestational age, } \\
\text { weeks), } M \text { (SD) }\end{array}$ & $40.5(2.1)$ & $40.9(1.4)$ \\
\hline $\begin{array}{l}\text { ICV at term-equivalent age } \\
\left(\mathrm{cm}^{3}\right), M(\mathrm{SD})\end{array}$ & $433.6(67.4)$ & $449.4(43.2)$ \\
\hline 7-Year characteristics & $\begin{array}{c}\mathrm{VP}(n=186) M \\
(\mathrm{SD})\end{array}$ & $\begin{array}{c}\text { Term }(n=37) M \\
(\mathrm{SD})\end{array}$ \\
\hline Age at assessment (years) & $7.5(0.3)$ & $7.6(0.2)$ \\
\hline
\end{tabular}

IQ and academic skills

$\begin{array}{lll}\text { Full IQ } & 97.2(13.8) & 109.2(12.7) \\ \text { Reading } & 99.0(18.3) & 109.1(18.3) \\ \text { Math computation } & 89.3(17.7) & 100.1(16.3)\end{array}$

Executive function

$\begin{array}{lcr}\text { Sustained attention } & 7.6(3.5) & 8.5(2.8) \\ \text { Verbal working memory } & 87.2(16.7) & 100.9(18.7) \\ \text { Goal setting } & 56.4(13.3) & 64.9(11.1)\end{array}$

Motor

$\begin{array}{lll}\text { Motor function } & 8.3(3.7) & 10.8(2.7)\end{array}$

Behavior

SDQ behavioral scale

$10.4(6.3)$

$6.5(5.2)$

$I C V$, intracranial volume; IQ, intelligence quotient; MRI, magnetic resonance imaging; SDQ, Strengths and Difficulties Questionnaire; VP, very preterm.

${ }^{a}$ Missing white matter injury severity data for $n=2$ controls.
Neonatal Basal Ganglia and Thalamic Volume Associations with 7-Year Neurodevelopment

Larger neonatal basal ganglia and thalamic volumes were associated with higher IQ and better academic achievement at age 7 years (Figure $3 a-c)$. These relationships weakened following adjustment for ICV. Neonatal basal ganglia and thalamic associations with IQ and academic achievement were generally similar in the VP and control groups (all $P$-values for interaction $>0.05$ ).

There was little evidence that neonatal basal ganglia and thalamic volumes were associated with executive functioning measures of sustained attention and working verbal memory (Figure 4a,b), before and after adjustment for ICV. There was some weak evidence that these volumes were positively associated with goal setting performance, but these associations diminished following adjustment for ICV (Figure 4c). Neonatal basal ganglia and thalamic volume associations with executive function measures were generally similar in the VP and control groups (all $P$-values for interaction $>0.05$ ) except for goal setting; larger thalamic volume was associated with better scores in the VP group before adjustment for ICV, but this association diminished after adjustment for ICV (interaction $P=0.043$, before adjusting for ICV; interaction $P=0.034$, after adjusting for ICV).

Larger neonatal basal ganglia volumes were associated with better motor functioning, and these relationships remained following adjustment for ICV (Figure 5a). Larger thalamic volume was associated with better motor function in the VP group only, with evidence of an inverse association in the control group (interaction $P<0.001$, before and after adjusting for ICV).

There was some evidence that smaller neonatal basal ganglia and thalamic volumes were associated with more general behavior problems (Figure $5 \mathbf{b}$ ) and these associations were generally similar in both the VP and control groups.

\section{DISCUSSION}

The current study shows that VP birth leads to underdevelopment or maldevelopment of individual components within the basal ganglia and thalamic network of the brain. Findings from this study support previous reports of smaller deep gray matter volumes in VP infants compared with termborn controls $(3,6,7)$, but also demonstrates that VP birth affects each individual nucleus within the basal ganglia. The current study further revealed selective relations between individual neonatal deep gray matter structure volumes and neurodevelopmental outcomes during later childhood, with these relationships partially explained by ICV or overall brain size.

Consolidation of thalamocortical connections only occurs in the third trimester of pregnancy, making these axonal connections vulnerable toward perinatal complications (hypoxia ischemia, inflammation, excitotoxicity, and free radical attack) (30). As the basal ganglia are relay structures that work in concert with the thalamus, injury toward the thalamocortical pathways probably also affects the basal 


\section{Articles | Loh et al.}
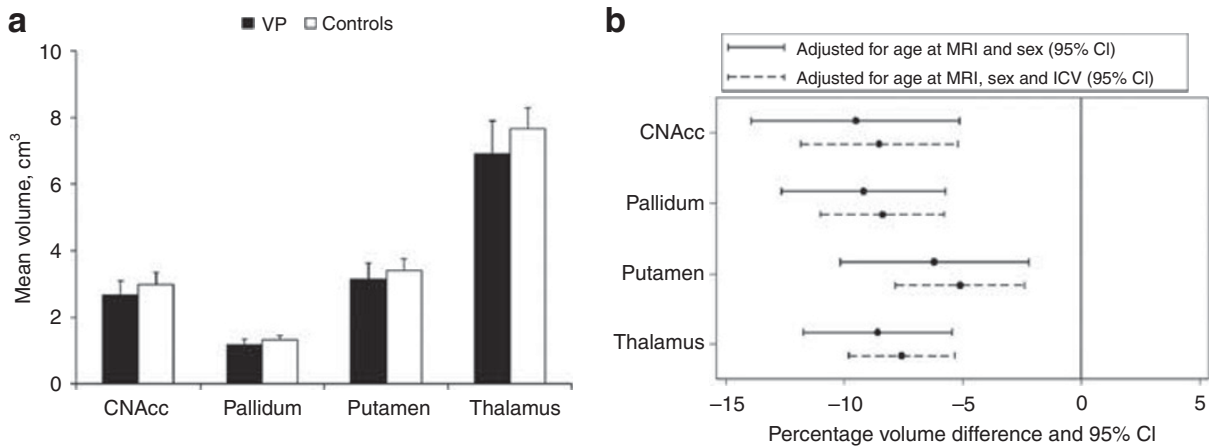

Figure 2. Very preterm (VP) infants vs. the term control group: (a) mean basal ganglia and thalamic volumes (error bars, SD) for the VP and control groups, (b) percentage volume differences (error bars, 95\% confidence intervals) in the basal ganglia and thalamus between VP infants compared with controls. Regression coefficient estimates of volume differences are presented as a percentage of the mean volume in the control group. All initial analyses (solid lines) adjusted for age at magnetic resonance imaging and sex, with secondary analyses (dashed lines) also adjusted for intracranial volume (ICV).

a
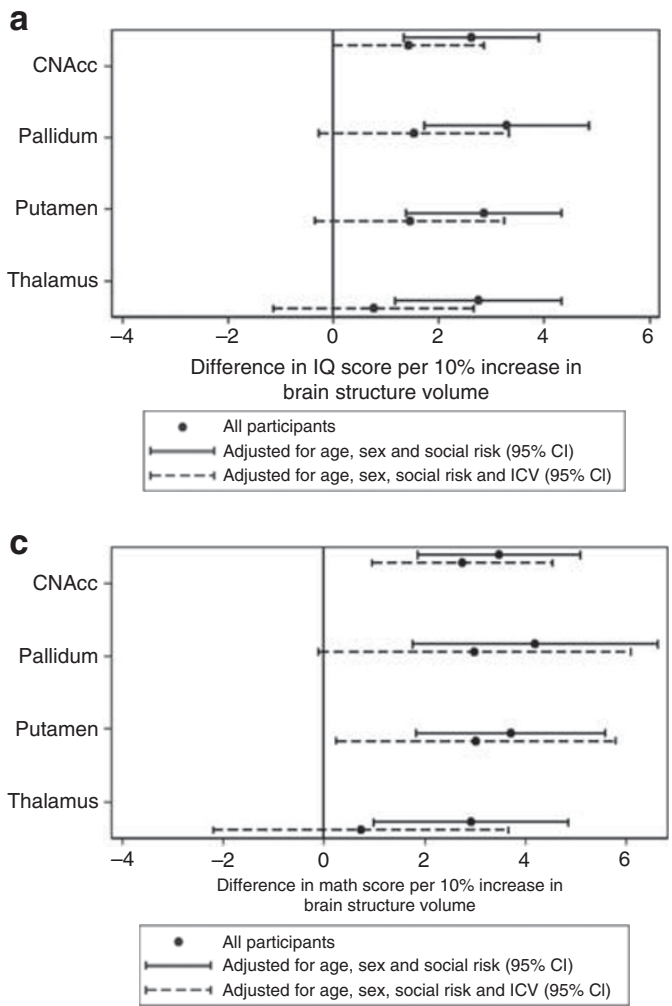

b

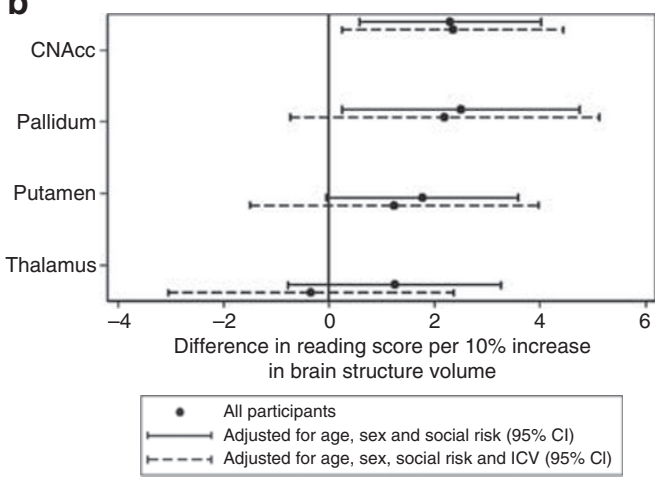

Figure 3. Neonatal basal ganglia and thalamic volumes in relation to cognitive and academic outcome at 7 years: (a) full IQ, (b) reading, and (c) math computation. Regression coefficient estimates represent the difference in outcome per $10 \%$ increase in brain volume, for the particular structure. Estimates (circle) incorporate data from all participants if group-by-volume interaction $P>0.05$; otherwise results are presented separately for control (square) and very preterm (VP, triangle) groups. All initial analyses (solid lines) are adjusted for age, sex and social risk (95\% Cl), with secondary analyses (dashed lines) also adjusted for intracranial volume (ICV).

ganglia. Smaller volumes in the basal ganglia and thalamus of VP infants compared with those in term-born infants may result from neuronal loss within the deep gray matter. These smaller volumes in VP infants compared with term-born infants may be related to white matter injury (WMI). Neuropathological examination has revealed neuronal loss in the basal ganglia and thalamus of preterm infants to be found exclusively in those with PVL (31), and another study demonstrated that volumetric deficits of these structures in preterm infants are exacerbated in the presence of severe WMI with focal lesions (7). These findings suggest that necrotic WMI may trigger retrograde axonal degeneration in the deep gray matter and, subsequently, neuronal degeneration (32). However, it has been shown that even preterm infants without 
a

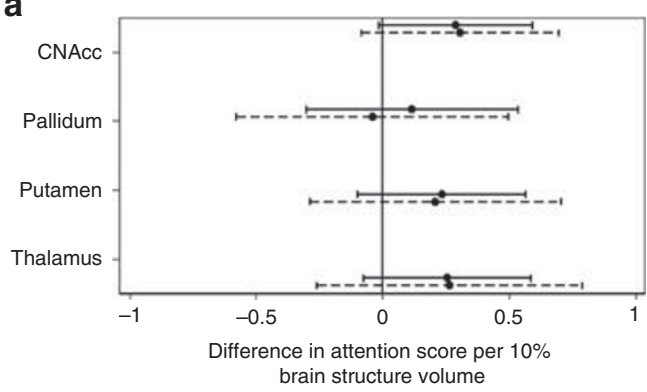

brain structure volume

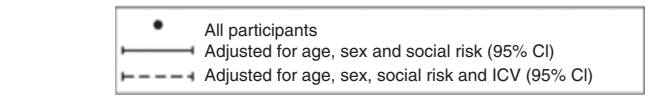

C

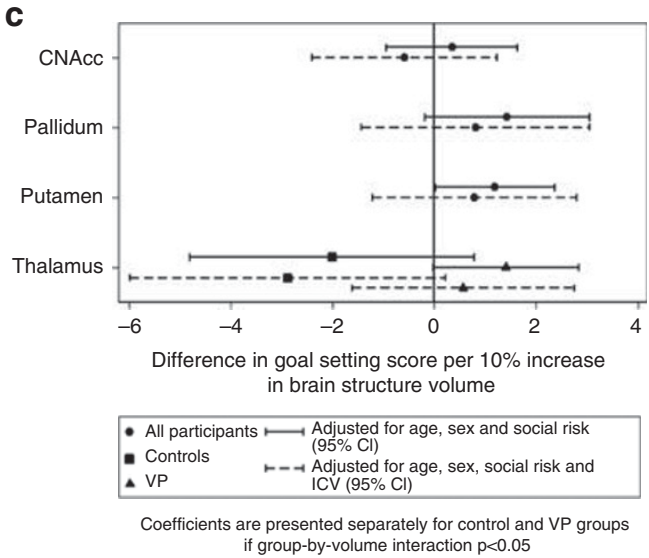

b

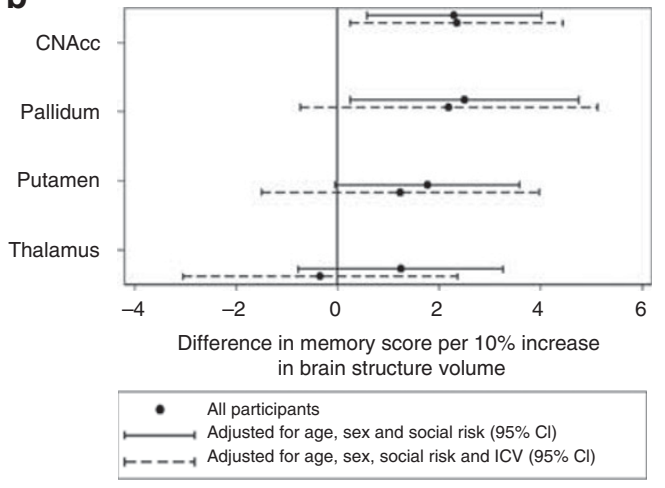

Figure 4. Neonatal basal ganglia and thalamic volume in relation to executive functioning outcome at 7 years: (a) sustained attention, (b) working verbal memory, and (c) goal planning. Regression coefficient estimates represent the difference in outcome per $10 \%$ increase in brain volume, for the particular structure. Estimates (circle) incorporate data from all participants if group-by-volume interaction $P>0.05$; otherwise results are presented separately for control (square) and very preterm (VP, triangle) groups. All initial analyses (solid lines) adjusted for age at assessment, sex, and social risk, with secondary analyses (dashed lines) also adjusted for intracranial volume (ICV).

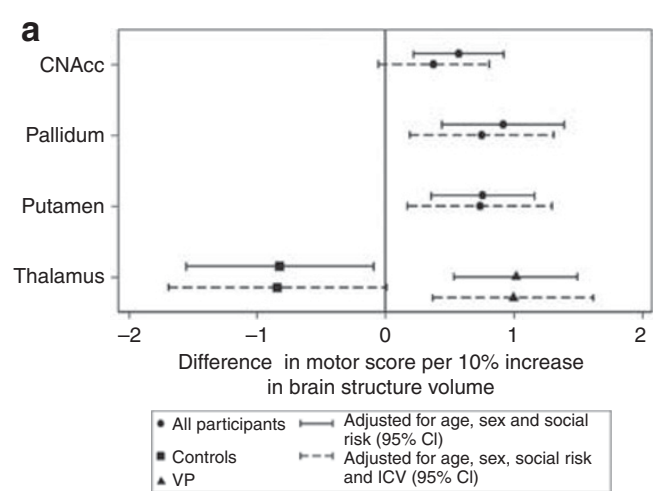

Coefficients are presented separately for control and VP groups if group-by-volume interaction $\mathrm{p}<0.05$

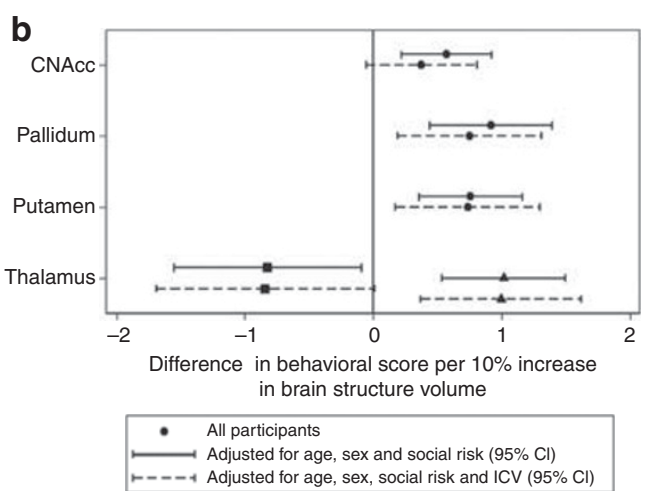

Adjusted for age, sex and social risk $(95 \% \mathrm{Cl})$
$1---1$ Adjusted for age, sex, social risk and ICV $(95 \% \mathrm{Cl})$

Figure 5. Neonatal basal ganglia and thalamic volume in relation to (a) motor and (b) behavioral outcome at 7 years. Regression coefficient estimates represent the difference in outcome per $10 \%$ increase in brain volume, for the particular structure. Estimates (circle) incorporate data from all participants if group-by-volume interaction $P>0.05$; otherwise results are presented separately for control (square) and very preterm (VP, triangle) groups. All initial analyses (solid lines) adjusted for age at assessment, sex, and social risk, with secondary analyses (dashed lines) also adjusted for intracranial volume (ICV).

severe WMI also have smaller basal ganglia and thalamic volumes compared with term-born infants (7). The current study comprises some VP infants with severe WMI, but the percentage of these infants is low (nine VP infants with cystic
PVL (4.3\%)), supporting the idea that reduced volumes are present even in preterm infants without severe WMI. Indeed, additional analyses exploring this idea by excluding VP infants with cystic PVL and grade 3 or 4 intraventricular hemorrhage 


\section{Articles | Loh et al.}

in this cohort revealed that volume reductions persisted (Supplementary Material S1 online). Neuropathological examination has shown that immature basal ganglia and thalamic neurons do not appear to degenerate in the presence of nonsevere WMI (diffuse WMI) (31). The mechanism for reduced deep gray matter volumes in VP infants without severe WMI may reflect a dysmaturation of the gray matter neurons (i.e., reduced complexity in dendritic arborization) rather than neuronal loss (32).

We have previously reported deep gray matter abnormality (MRI signal abnormality and/or reduced cross-sectional area) at term-equivalent age to be associated with poorer 7-year cognitive outcome in memory and learning performance using the same cohort as the current study (33). We extend those qualitative findings by demonstrating that VP infants have smaller quantitative basal ganglia and thalamic volumes compared with controls at term-equivalent age, and that smaller volumes are associated with poorer IQ and academic outcomes at age 7 years, with these relationships partially explained by ICV. A study that concurrently assessed the relationship between basal ganglia volume and IQ in the caudate nucleus of VP 7-year-old children also reported a positive relationship between brain volume and IQ (34). Basal ganglia and thalamic volume associations with IQ and academic outcomes may be explained by their role in motivation and learning. The striatum, which comprises the CNAcc and putamen, is densely innervated by dopaminergic neurons. Dopamine is the neurotransmitter for striatal neurons, and a larger striatal volume (particularly the caudate nucleus) may reflect an increased distribution of dopamine receptors (35) leading to a behavior that seeks the motivational value in learning.

There was little evidence that neonatal basal ganglia and thalamic volumes were related to executive function, except for some weak evidence in goal setting performance, but this relationship diminished after adjustment for ICV. Executive function is a set of higher-order cognitive skills, which involves attentional control, information processing, goal setting, and cognitive flexibility, and it is commonly reported to be impaired in VP survivors (1). Despite the cortico-basal ganglia-thalamo-cortical network being often implicated in executive functioning, there was not much evidence from the current study that neonatal basal ganglia and thalamic volumes were associated with specific executive functioning domains. It has been demonstrated that local morphometric changes from shape analyses may be more strongly related to cognitive functioning than widespread volume changes in healthy controls (36). Therefore, regional changes within these basal ganglia and thalamic structures rather than global volumes may be more sensitive in detecting more complex brain structurefunction relationships, such as executive functioning.

In the current study, smaller neonatal basal ganglia and thalamic volumes at term-equivalent age were associated with poorer motor function at age 7 years, before and after adjusting for ICV. Our findings are in keeping with a Finnish study that demonstrated smaller basal ganglia and thalamic volumes at term-equivalent age to be associated with poorer neuromotor outcome at age 11 years in $98 \mathrm{VP}$ children (11). It is well-accepted that the basal ganglia and thalamus are highly connected with the premotor, motor, and somatosensory cortices (37), and the current study revealed brain volumemotor outcome associations in these structures. Using diffusion MRI, thalamic connectivity with the motor cortex has been shown to decrease following preterm birth (38). However, examining cortico-basal ganglia connectivity in relation to motor outcome within preterm populations is yet to be studied, and this might be an important area of future research given the current study's findings.

The current study demonstrated that larger neonatal basal ganglia and thalamic volumes were generally related to less behavioral problems. Behavioral areas of concern in children born preterm include internalizing problems, fewer adaptive skills, increased attentional problems, increased hyperactivity, and poorer social competency (39). Using diffusion MRI, a United States study has demonstrated decreased structural connectivity within the cortico-basal ganglia-thalamo-cortical pathways of preterm children to be associated with poorer performance on the SDQ scale (40). Interestingly, that same study showed decreased structural connectivity to be most strongly associated with increased hyperactivity as measured by the SDQ (40). This suggests that abnormality in the basal ganglia and thalamus may relate to behavioral problems in terms of hyperactivity within preterm children.

Instead of the expected positive relationship between brain volume and functional outcome in the control group, there was some evidence of a negative relationship, particularly for the putamen and thalamus in goal setting, motor, and behavioral measures. These unexpected findings may reflect limited power in the sample size of the control group (37 controls vs. $186 \mathrm{VP}$ children). Alternatively, these negative relationships may reflect the lack of sensitivity for brain volume as a marker of brain function at certain stages of development, considering that the basal ganglia and thalamus undergo both periods of expansion and contraction with age (41).

The strength of this study is that it contributes to the limited literature examining brain volumes at term-equivalent age in association with the long-term neurodevelopmental outcomes. Although exploratory, the current study showed some evidence that neonatal basal ganglia and thalamic volumes were associated with 7-year neurodevelopmental outcomes, particularly for motor, IQ, and academic achievement. Although these relationships weakened following adjustment for ICV, this is expected as the basal ganglia and thalamus are brain relay structures that are highly connected with other parts of the brain including the cortex and white matter, which constitute a large proportion of the brain. A limitation of this study is that we did not explore whether comorbidities of prematurity mediated the smaller basal ganglia and thalamic volumes in VP infants compared with term-born infants at term-equivalent age. Given that there was evidence of these smaller deep gray matter volumes being associated with worse neurodevelopmental outcomes, it 
is important for future research to identify potentially modifiable factors that may improve the long-term outcome of VP infants.

Segmentation of deep gray matter structures is challenging in the neonatal brain. Visualization of the basal ganglia and thalamus on MR images is different between a neonate and an adult, because of different tissue contrasts resulting from myelination taking place during the neonatal period. Despite this, the current study produced a reliable custom-made neonatal template to automatically segment the basal ganglia and thalamus using a previously validated technique. Furthermore, all automatically generated segmentations were inspected and manually edited, if necessary, to ensure the greatest possible accuracy.

\section{CONCLUSIONS}

This study showed that VP birth affects all basal ganglia nuclei, as well as the thalamus at term-equivalent age, suggesting that VP birth leads to neuronal loss and/or neuronal dysmaturation within these deep gray matter structures. Smaller neonatal basal ganglia and thalamic volumes were associated with a range of long-term neurodevelopmental impairments at age 7 years, being most strongly associated with motor functioning, IQ, and academic achievements.

\section{SUPPLEMENTARY MATERIAL}

Supplementary material is linked to the online version of the paper at http://www.nature.com/pr

\section{ACKNOWLEDGMENTS}

We are grateful for the help and support of the Victorian Infant Brain Studies and Developmental Imaging groups, as well as the Melbourne Children's MRI Centre at the Murdoch Childrens Research institute. We also thank the families and children who participated in this study.

\section{AUTHOR CONTRIBUTIONS}

W.Y.L. contributed to the study design and conceptualization, analyzed and interpreted the data, drafted the initial manuscript, and approved the final manuscript as submitted; P.J.A., J.L.Y.C., A.J.S., T.E.I, A.C., L.W.D., and D. K.T. contributed to the study design and conceptualization, interpreted the data, reviewed and revised the manuscript, and approved the final manuscript as submitted; J.C., C.M., and K.J.L. contributed to the data analysis and interpretation, reviewed and revised the manuscript, and approved the final manuscript as submitted.

\section{STATEMENT OF FINANCIAL SUPPORT}

This study was supported by Australia's National Health \& Medical Research Council: Centre for Clinical Research Excellence 546519 to L.W.D. and P.J.A.; Centre for Research Excellence 1060733 to L.W.D., P.J.A., J.L.Y.C., D.K.T., A.J.S., and W.Y.L.; Project Grants 237117 to L.W.D., 491209 to P.J.A.; Senior Research Fellowship 1081288 to P.J.A.; Career Development Fellowships 1108714 to A.J.S., 1085754 to D.K.T.; Early Career Fellowship 1053787 to J.L.Y.C.. This study was also supported by the National Institutes of Health (HD058056), the Victorian Government's Operational Infrastructure Support Program, and The Royal Children's Hospital Foundation.

Disclosure: The authors declare no conflict of interest.

\section{REFERENCES}

1. Aarnoudse-Moens CSH, Weisglas-Kuperus N, van Goudoever JB, Oosterlaan J. Meta-analysis of neurobehavioral outcomes in very preterm and/or very low birth weight children. Pediatrics 2009;124:717-28.
2. de Kieviet JF, Piek JP, Aarnoudse-Moens CS, Oosterlaan J. Motor development in very preterm and very low-birth-weight children from birth to adolescence: a meta-analysis. JAMA 2009;302:2235-42.

3. Inder TE, Warfield SK, Wang H, Hüppi PS, Volpe JJ. Abnormal cerebral structure is present at term in premature infants. Pediatrics 2005;115: 286-94.

4. Arsalidou M, Duerden EG, Taylor MJ. The centre of the brain: topographical model of motor, cognitive, affective, and somatosensory functions of the basal ganglia. Hum Brain Mapp 2013;34:3031-54.

5. Alexander GE, DeLong MR, Strick PL. Parallel organization of functionally segregated circuits linking basal ganglia and cortex. Annu Rev Neurosci 1986;9:357-81.

6. Boardman JP, Counsell SJ, Rueckert D, et al. Abnormal deep grey matter development following preterm birth detected using deformation-based morphometry. Neuroimage 2006;32:70-8.

7. Srinivasan L, Dutta R, Counsell SJ, et al. Quantification of deep gray matter in preterm infants at term-equivalent age using manual volumetry of 3-tesla magnetic resonance images. Pediatrics 2007;119:759-65.

8. Lind A, Parkkola R, Lehtonen L, et al. Associations between regional brain volumes at term-equivalent age and development at 2 years of age in preterm children. Pediatr Radiol 2011;41:953-61.

9. Young JM, Powell TL, Morgan BR, et al. Deep grey matter growth predicts neurodevelopmental outcomes in very preterm children. Neuroimage 2015;111:360-8.

10. Roberts G, Anderson PJ, Doyle LW, Victorian Infant Collaborative Study Group. The stability of the diagnosis of developmental disability between ages 2 and 8 in a geographic cohort of very preterm children born in 1997. Arch Dis Child 2010;95:786-90.

11. Setänen S, Lehtonen L, Parkkola R, Aho K, Haataja L, PIPARI Study Group. Prediction of neuromotor outcome in infants born preterm at 11 years of age using volumetric neonatal magnetic resonance imaging and neurological examinations. Dev Med Child Neurol 2016;58:721-7.

12. Thompson DK, Ahmadzai ZM, Wood SJ, et al. Optimizing hippocampal segmentation in infants utilizing MRI post-acquisition processing. Neuroinformatics 2012;10:173-80.

13. Loh WY, Connelly A, Cheong JL, et al. A new MRI-based pediatric subcortical segmentation technique (PSST). Neuroinformatics 2016;14: 69-81.

14. Avants BB, Epstein CL, Grossman M, Gee JC. Symmetric diffeomorphic image registration with cross-correlation: evaluating automated labeling of elderly and neurodegenerative brain. Med Image Anal 2008;12:26-41.

15. Avants BB, Yushkevich P, Pluta J, et al. The optimal template effect in hippocampus studies of diseased populations. Neuroimage 2010;49: 2457-66.

16. Spittle AJ, Thompson DK, Brown NC, et al. Neurobehaviour between birth and 40 weeks' gestation in infants born $<30$ weeks' gestation and parental psychological wellbeing: predictors of brain development and child outcomes. BMC Pediatr 2014;14:111.

17. Walsh JM, Doyle LW, Anderson PJ, Lee KJ, Cheong JL. Moderate and late preterm birth: effect on brain size and maturation at term-equivalent age. Radiology 2014;273:232-40.

18. Beare R, Chen J, Kelly C, et al. Neonatal brain tissue classification with morphological adaptation and unified segmentation. Front Neuroinform 2016;10:12.

19. Kuklisova-Murgasova M, Aljabar P, Srinivasan L, et al. A dynamic 4D probabilistic atlas of the developing brain. Neuroimage 2011;54:2750-63.

20. Ashburner J, Friston KJ. Unified segmentation. Neuroimage 2005;26: 839-51.

21. Wechsler D. Wechsler Abbreviated Scale of Intelligence (WASI). New York, NY: The Psychological Corporation, 1999.

22. Wilkinson GS, Robertson GJ. Wide Range Achievement Test (WRAT4). Lutz, FL: Psychological Assessment Resources, 2006.

23. Manly T, Anderson V, Nimmo-Smith I, Turner A, Watson P, Robertson IH. The differential assessment of children's attention: the Test of Everyday Attention for Children (TEA-Ch), normative sample and ADHD performance. J Child Psychol Psychiatry 2001;42:1065-81. 


\section{Articles | Loh et al.}

24. Pickering S, Gathercole S. Working Memory Test Battery for ChildrenManual. London: The Psychological Corporation, 2001.

25. Gioia G, Isquith P, Guy S, Kenworthy L. BRIEF: Behavior Rating Inventory of Executive Function. Psychological Assessment Resources, Lutz, FL, 2000.

26. Henderson SE, Sugden DA, Barnett AL. Movement Assessment Battery for Children-2 (Movement ABC-2), 2nd edn. London, UK: The Psychological Corporation, 2007.

27. Goodman R. The strengths and difficulties questionnaire: a research note. J Child Psychol Psychiatry 1997;38:581-6.

28. Treyvaud K, Aldana AC, Scratch SE, et al. The influence of multiple birth and bereavement on maternal and family outcomes 2 and 7 years after very preterm birth. Early Hum Dev 2016;100:1-5.

29. Peterson BS, Vohr B, Staib LH, et al. Regional brain volume abnormalities and long-term cognitive outcome in preterm infants. JAMA 2000;284: 1939-47.

30. Volpe JJ, Kinney HC, Jensen FE, Rosenberg PA. Review: Reprint of "The developing oligodendrocyte: key cellular target in brain injury in the premature infant". Int J Dev Neurosci 2011;29:565-82.

31. Pierson C, Folkerth R, Billiards S, et al. Gray matter injury associated with periventricular leukomalacia in the premature infant. Acta Neuropathol 2007;114:619-31.

32. Back SA, Miller SP. Brain injury in premature neonates: a primary cerebral dysmaturation disorder? Ann Neurol 2014;75:469-86.

33. Omizzolo C, Scratch SE, Stargatt R, et al. Neonatal brain abnormalities and memory and learning outcomes at 7 years in children born very preterm. Memory 2013;22:605-15.
34. Abernethy LJ, Cooke RWI, Foulder-Hughes L. Caudate and hippocampal volumes, intelligence, and motor impairment in 7-year-old children who were born preterm. Pediatr Res 2004;55:884-93.

35. Woodward ND, Zald DH, Ding Z, et al. Cerebral morphology and dopamine D2/D3 receptor distribution in humans: a combined [18F] fallypride and voxel-based morphometry study. Neuroimage 2009; 46:31-8.

36. Sandman CA, Head K, Muftuler LT, Su L, Buss C, Davis EP. Shape of the basal ganglia in preadolescent children is associated with cognitive performance. Neuroimage 2014;99:93-102.

37. Draganski B, Kherif F, Klöppel S, et al. Evidence for segregated and integrative connectivity patterns in the human basal ganglia. J Neurosci 2008;28:7143-52.

38. Ball G, Boardman JP, Aljabar P, et al. The influence of preterm birth on the developing thalamocortical connectome. Cortex 2013;49:1711-21.

39. Farooqi A, Hägglöf B, Sedin G, Gothefors L, Serenius F. Mental health and social competencies of 10- to 12-year-old children born at 23 to 25 weeks of gestation in the 1990s: a Swedish national prospective followup study. Pediatrics 2007;120:118-33.

40. Fischi-Gomez E, Vasung L, Meskaldji DE, et al. Structural brain connectivity in school-age preterm infants provides evidence for impaired networks relevant for higher order cognitive skills and social cognition. Cereb Cortex 2015;25:2793-805.

41. Raznahan A, Shaw PW, Lerch JP, et al. Longitudinal four-dimensional mapping of subcortical anatomy in human development. Proc Natl Acad Sci USA 2014;111:1592-7. 